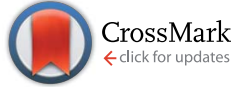

Cite this: RSC Adv., 2015, 5, 60804

\section{Artificial inverted compound eye structured polymer films with light-harvesting and self- cleaning functions for encapsulated III-V solar cell applications $\dagger$}

\begin{abstract}
Jung Woo Leem and Jae Su Yu*
We report the artificial inverted compound eye structured (ICESs) polydimethylsiloxane (PDMS) films with lightharvesting and self-cleaning functions for the enhancement of solar power generation in encapsulated III-V gallium arsenide (GaAs) single-junction solar cells. The ICESS PDMS films are fabricated by facile, simple, and cost-effective soft lithography using sapphire master molds with the CESs consisting of hierarchical nanotextures/periodic microgratings prepared by thermally dewetted gold nanopatterning and subsequent dry etching processes. By attaching the ICESs PDMS film with a hydrophobic surface (i.e., water contact angle $\left(\theta_{C A}\right)$ of $\sim 121^{\circ}$ ) to the coverglass, the total and diffuse transmittances of the coverglass are simultaneously increased over a wide wavelength range of 350-900 nm, exhibiting higher solar weighted transmittance (SWT) of $\sim 94.3 \%$ and average haze ratio $\left(H_{\text {avg }}\right)$ of $\sim 67.6 \%$ than those of the bare coverglass (i.e., $\theta_{\mathrm{CA}} \approx 32^{\circ}$, SWT $\approx 90.2 \%$, and $H_{\mathrm{avg}} \approx 4.1 \%$, respectively). The resulting encapsulated III-V GaAs singlejunction solar cells with the ICESs PDMS/coverglass show an enhanced power conversion efficiency (PCE) of $24.3 \%$ compared to the encapsulated solar cell with the bare coverglass (i.e., PCE $=22.96 \%$ ) due to the increased short circuit current density from 26 to $27.64 \mathrm{~mA} \mathrm{~cm}^{-2}$, indicating the PCE increment percentage of $\sim 5.8 \%$. Moreover, it also exhibits superior device performance at varying angles of incident light. For the long-term self-cleaning effect on the device efficiency, there is no significant variation in the PCE after approximately one month, indicating a low PCE drop percentage of $\sim 1.4 \%$.
\end{abstract}

Received 3rd April 2015 Accepted 9th July 2015 DOI: $10.1039 / \mathrm{c} 5 \mathrm{ra05991g}$ www.rsc.org/advances drawbacks including durability issues, thermal expansion mismatch, poor or reduced adhesion on certain substrates, sensitivity to thickness variations, and material selection as well as narrow low reflection band for incident wavelengths and angles, ${ }^{8-10}$ may not be suitable under real harsh environments. As an alternative of conventional antireflection layers, over the past years, there have been many studies on the efficiency enhancement of solar cells with textured surfaces (i.e., biomimetic micro- or nanostructures) for efficient antireflection and light scattering. ${ }^{11-15}$ Particularly, artificial compound eye structures (CESs) inspired from corneas of insects, which are usually composed of thousands of microstructures covered with nanonipple arrays, effectively reduce the surface reflection (or enhance the transmission) over wide ranges of incident wavelengths and angles because of the formation of the effective gradient-refractive-index profile between air and the bulk surface. ${ }^{\mathbf{1 6 - 2 2}}$ Besides, the CESs with microscale periods can also extend the effective optical paths and promote the transmitted diffuse lights, while keeping high total transmission properties (e.g., high optical haze).$^{23-26}$ In addition, due to this extension of optical paths, the microstructures (e.g., microlenses, microcones) can help to reduce total internal reflection losses in optoelectronic devices like light-emitting diodes. ${ }^{27,28}$
Department of Electronics and Radio Engineering, Institute for Wearable Convergence
Electronics, Kyung Hee University, 1732 Deogyeong-daero, Giheung-gu, Yongin-si
446-701, South Korea. E-mail: jsyu@khu.ac.kr
$\dagger$ Electronic supplementary information (ESI) available: SEM images of the
thermally dewetted Au nanopatterns for flat sapphire and p-MGs/sapphire,
optical measurement system with an integrating sphere, photograph of a water
droplet for the flat bare PDMS film, schematic diagram for the water droplet
cleaning concept, and photograph of the ICESs PDMS film laminated on the
coverglass. See DOI: $10.1039 / \mathrm{c} 5 \mathrm{ra} 05991 \mathrm{~g}$

\section{Introduction}

hently, there has been orow crisis and environmental issues. To effectively harvest sunlight in solar cells, an efficient antireflection layer, which can material-based thin-film antireflection layers such as magnesium fluorides and porous silicon oxides, which have some 
On the other hand, polydimethylsiloxane (PDMS) is widely used as conformable and elastomeric replica stamps, substrates, or membranes in the soft lithography, which is relatively a more simple, large-scalable, and cost-effective micro- and nanopatterning technique with high-throughput production compared to the energy-intensive patterning methods such as electron-beam lithography, nanoimprint lithography, and photolithography, owing to its low free surface energy, flexibility, transparency, and hardness. ${ }^{29-33}$ Furthermore, the PDMS can be suitable as an antireflective protection layer of the coverglass in encapsulated solar cells because of its lower refractive index of 1.4-1.43 (i.e., $n \approx 1.5-1.52$ for glass). ${ }^{32-35}$ Also, the cured PDMS film attached on other flat substrates such as wafers and glasses has shown a very good and strong adhesion property due to the mainly van der Waals force (e.g., adhesive strengths of $\sim 180$ $\mathrm{kPa}){ }^{36,37}$ Therefore, the PDMS film can not only be powerfully well-laminated on the flat surface of coverglasses, but also reversibly easily-detached from it. From these reasons, as an antireflective protection layer of coverglasses, the compound eye structured PDMS film with high optical transparency and haze can boost the light harvesting in the underneath encapsulated solar cells, resulting in the efficiency improvement of devices. In addition, for photovoltaic industry applications, mass production would be also possible because the large-scale fabrication techniques of micro- and nanostructured polymer films have been developed by roll-to-roll or roll-to-plate processes using the soft imprint lithography. ${ }^{\mathbf{3 8 - 4 0}}$ Meanwhile, the roughened structures could enhance the surface hydrophobicity of materials, related to the Cassie-Baxter wetting behavior. ${ }^{\mathbf{4 1 , 4 2}}$ This hydrophobic surface, which can self-clean the dust particles and contaminants on the surface, is exceedingly useful in practical solar cell applications..$^{35,43,44}$ Thus, it is very meaningful to analyze the optical properties and water wettability by applying the hierarchical nano/microstructures into the surface of PDMS films as the antireflective protection layer of coverglasses for efficiency improvement of encapsulated solar cells. In this work, we fabricated the artificial inverted CESs (ICESs) on the surface of PDMS films by the soft lithography method using sapphire master molds with the CESs prepared by thermally dewetted gold $(\mathrm{Au})$ nanopatterning and dry etching processes. Their water wetting behaviors as well as optical properties were studied. The effect of the ICESs PDMS film as an antireflection layer of coverglasses on the device characteristics of encapsulated gallium arsenide (GaAs) single-junction solar cells was investigated. For a theoretical analysis of optical light scattering properties, the finite-difference time-domain (FDTD) simulation was performed. Besides, the incident light angle-dependent device performance and the long-term device stability for demonstrating the self-cleaning function were also explored.

\section{Experimental and numerical modeling details}

\section{Fabrication of ICESs PDMS films}

Fig. 1 shows schematic illustrations of (a) fabrication process steps for (i) CESs on the master mold by thermally dewetted Au nanopatterns and dry etching and (ii) ICESs on the PDMS film using the CESs patterned mold by the soft lithography method and (b) encapsulated III-V GaAs single-junction solar cell structure with the ICESs PDMS film/coverglass. For the master mold with CESs, the patterned sapphire substrates, which were purchased from AND Corporation, consisting of conical periodic microgratings ( $\mathrm{p}-\mathrm{MGs}$ ) with two-dimensional hexagonal pattern arrays, having the average height and period of $\sim 1.5 \pm$ $0.1 \mu \mathrm{m}$ and $\sim 2.5 \pm 0.1 \mu \mathrm{m}$, respectively, were used. For the formation of closely-packed nanotextures (NTs) with periods smaller than $200 \mathrm{~nm}$, the Au thin film with a thickness of $4 \mathrm{~nm}$ was deposited on the p-MGs/sapphire substrate by a thermal evaporation system. And then, the samples were heat-treated by using a rapid thermal annealing system at a temperature of $600{ }^{\circ} \mathrm{C}$ for $2 \mathrm{~min}$ in a nitrogen environment. In this case, the $\mathrm{Au}$ thin film was agglomerated into the nanosized particles due to its increased surface energy by the heat (see Fig. S1 in the ESI $\dagger) .{ }^{16,45}$ Using the Au nanopatterns as an etch mask, the NTs on the surface of p-MGs/sapphire substrates were fabricated, thus creating the compound eye structures (CESs), as shown in Fig. 1(a)(i). The dry etching was performed with $50 \mathrm{~W}$ of RF power and $200 \mathrm{~W}$ of additional ICP power at $3 \mathrm{mTorr}$ of process pressure for $7 \mathrm{~min}$ in $\mathrm{Ar} / \mathrm{Cl}_{2} / \mathrm{BCl}_{3}$ (2:1:4) plasma. The remaining Au was removed using the etchant solution based on potassium iodide and iodine $\left(\mathrm{KI} / \mathrm{I}_{2}\right)$. To fabricate the ICESs on the surface of PDMS films, as shown in Fig. 1(a)(ii), a hard PDMS, which was often used to prevent the deformation and distortion of replica nanofeatures, ${ }^{46}$ was prepared by mixing a $3.4 \mathrm{~g}$ of trimethylsiloxyterminated vinylmethylsiloxanedimethylsiloxane (VDT-731; Gelest, Inc.), one drop of a platinum catalyst (platinum divinyltetramethyldisiloxane, SIP6831.2; Gelest, Inc.), and two drops of a modulator (1,3,5,7tetravinyl-1,3,5,7-tetramethylcyclotetrasiloxane, SIT 7900.0; Gelest, Inc.). After that, the methylhydrosiloxanedimethylsiloxane (HMS-301; Gelest, Inc.) was added into this mixture with subsequent stirring for 2 min and degassing for 5 min in the vacuum desiccators. Afterwards, the hard PDMS was spin-coated on the fabricated master molds with CESs at 1000 rpm for $40 \mathrm{~s}$ and $500 \mathrm{rpm}$ for $5 \mathrm{~min}$, sequentially, followed by curing at a temperature of $75{ }^{\circ} \mathrm{C}$ for $25 \mathrm{~min}$ in an oven. As a nondestructive and reversible manner, to enable manual application and intimate conformable contact on flat surfaces, after degassing the Sylgard 184 (Dow Corning Co.) polymer with a ratio of $10: 1$ (base : agent) for $1 \mathrm{~h}$, which is the soft PDMS, it was poured on the hard PDMS/master molds, and then the samples were cured at $75^{\circ} \mathrm{C}$ for $2 \mathrm{~h}$ in an oven. Finally, the PDMS films (hard/soft PDMS) were carefully separated from the master molds, which resulted in the inverted compound eye structured (ICESs) PDMS films with a size of $2 \times$ $2 \mathrm{~cm}^{2}$ and a thickness of $\sim 300 \mu \mathrm{m}$. For comparison, the inverted NTs (INTs) PDMS film was also fabricated using the flat sapphire substrate with NTs by the same fabrication process. For the epitaxial growth of GaAs single-junction solar cell structure, an AIXTRON multi-wafer metal organic chemical vapor deposition reactor was used on $2^{\circ}$ misoriented n-type GaAs substrate from (100) towards (111) direction. The GaAs single-junction solar cells with a chip aperture area of 
$0.3025 \mathrm{~cm}^{2}$ including metal contact grids were fabricated by conventional photolithography, metal evaporation, lift-off, and etch processes. And then, the cells were integrated on metalcoated alumina-based printed circuit boards by bonding the top $\mathrm{Au}$ wires and the rear silver paste. The cells on printed circuit boards were completely encapsulated with an ultraviolet curable adhesive (NOA 89, Norland Products Inc.) and a costeffective soda lime float coverglasses (Microscope slides, Sail brand, Cat. no. 7101, manufactured by Yancheng Huida medical instruments Co., China) with a size of $1 \times 1 \mathrm{~cm}^{2}$. The NOA89 adhesive was poured onto the cells bonded on printed circuit boards and subsequently covered by the coverglass, followed by exposing an ultraviolet source, which is a $40 \mathrm{~W}$-mercury ultraviolet lamp with a light emission wavelength range of $297-435 \mathrm{~nm}$, for $20 \mathrm{~min}$ to cure the NOA 89 adhesive. As can be seen in Fig. 1(b), to improve the light harvesting in the underneath cell absorption layer, the fabricated ICESs PDMS films with a size of $1 \times 1 \mathrm{~cm}^{2}$ were laminated on the coverglass of encapsulated III-V GaAs singlejunction solar cells as an antireflective protective layer.

\section{Characterization instruments}

Field-emission scanning electron microscope (FE-SEM; LEO SUPRA 55, Carl Zeiss) and atomic force microscope (AFM; D3100, Veeco) measurements were carried out to investigate surface morphologies and patterned profiles of the fabricated samples. The optical transmittance and reflectance properties were characterized by using a UV-vis-NIR spectrophotometer (Cary 5000, Varian) with an integrating sphere (see Fig. S2 in the ESI $\dagger$ ). The water contact angles were measured and averaged at three different positions on the surface of samples by using a contact angle measurement system (Phoenix-300, SEO Co., Ltd) with $\sim 5 \mu \mathrm{L}$ droplets of de-ionized water at room temperature. A solar simulator (WXS-220S-L2, Wacom) was used for currentvoltage measurements of devices under 1-sun air mass 1.5 global illumination. The external quantum efficiency (EQE) was characterized by using a QEX7 system (PV Measurements, Inc.).

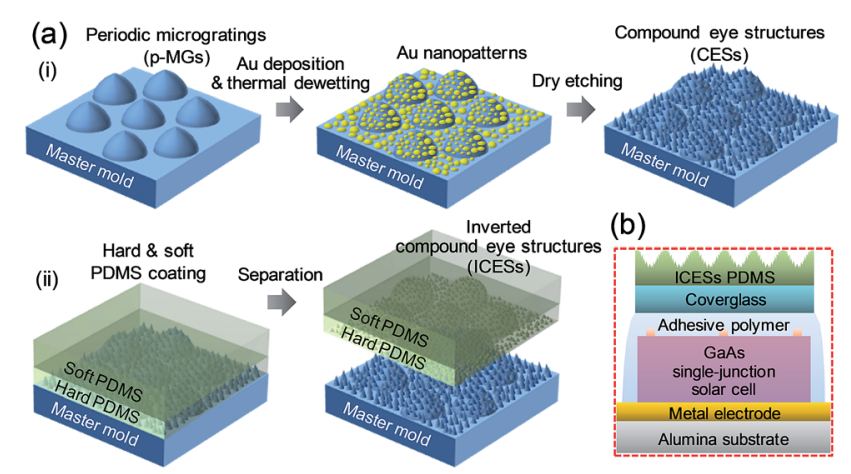

Fig. 1 Schematic illustrations of (a) fabrication process steps for (i) CESs on the master mold by thermally dewetted Au nanopatterns and dry etching and (ii) ICESs on the PDMS film using the CESs patterned mold by the soft lithography method and (b) encapsulated III-V GaAs single-junction solar cell structure with the ICESS PDMS film/ coverglass.

\section{Numerical modelling and simulations}

The theoretical calculation on an optical light scattering behavior of the ICES PDMS film was performed using the FDTD method. For the FDTD optical simulations, a commercial software (FullWAVE, Rsoft Design Group) was utilized. ${ }^{47}$ To design the theoretical model, in calculations, the hierarchical inverted nanotextures (nanocones)/periodic microgratings on the surface of PDMS film were represented by a periodic geometry in the Cartesian coordinate system by a scalar-valued function of two variables, $f(x, z)$, for simplicity. It was assumed that the incident light entered from air into the structure at normal incidence. The amplitude of $y$-polarized electric field $\left(E_{y}\right)$ was calculated for the incident plane wave with a slab mode beam profile in the continuous-wave mode, which was normalized at a wavelength $(\lambda)=532 \mathrm{~nm}$. Here, perfectly-matched-layer boundary conditions were enforced. The simulation time step and time were set to be $3 \mathrm{~nm}$ per c and $300 \mu \mathrm{m}$ per $\mathrm{c}$, where $\mathrm{c}$ is the speed of light in vacuum, respectively, with a grid size of $5 \mathrm{~nm}$. For the PDMS, the refractive index was assumed to be 1.43 while the extinction coefficient was not considered because it can be ignored.

\section{Results and discussion}

\section{Surface morphologies and patterned profiles}

Fig. 2 shows the FE-SEM images of (a) sapphire master molds with (i) the flat NTs, (ii) the p-MGs, and (iii) the CESs and (b) PDMS films with inverted patterns of the corresponding master molds. For the PDMS films with (i) the flat INTs, (ii) the p-IMGs, and (iii) the ICESs in Fig. 2(b), AFM images are also shown in Fig. 2(c). As shown in Fig. 2(a), the NTs, p-MGs, and CESs (i.e., hierarchical NTs/p-MGs) were well formed on the surfaces of corresponding master molds, respectively. By using the soft lithography, the micrograting arrayed patterns on sapphire master molds were negatively well transferred into the surface of PDMS films without any deformation and distortion, as shown in Fig. 2(b)(ii) and (iii). However, the nanotextured patterns on the master mold with the average height of $\sim 180 \pm$ $20 \mathrm{~nm}$ were poorly copied on the surface of PDMS film in spite of the use of hard PDMS. This may be attributed to the very closely-packed patterns of NTs (i.e., high density of $\sim 70 \%$ ) with the low average period of $\sim 100 \pm 20 \mathrm{~nm}$, and thus the hard PDMS solution could not wedge into the empty space between the NTs. As a result, the INTs with a low average depth of $\sim 90 \pm$ $10 \mathrm{~nm}$ were formed on the surfaces of flat and periodic inverted microgratings (p-IMGs) PDMS films, as can be seen in Fig. 2(b)(i) and (iii). Nevertheless, the relatively favorable INTs, p-IMGs, and ICESs patterns were formed on the surface of PDMS films from the corresponding master molds, respectively, by the soft lithography pattern transfer method, which can be also confirmed in the AFM images of Fig. 2(c).

\section{Surface wettability and self-cleaning behaviors}

The dust particles on the surface of coverglass can be harmful to the performance of encapsulated solar cells under real outdoor environments. $^{35,48}$ Therefore, it is necessary to employ the 
(a)

(b)
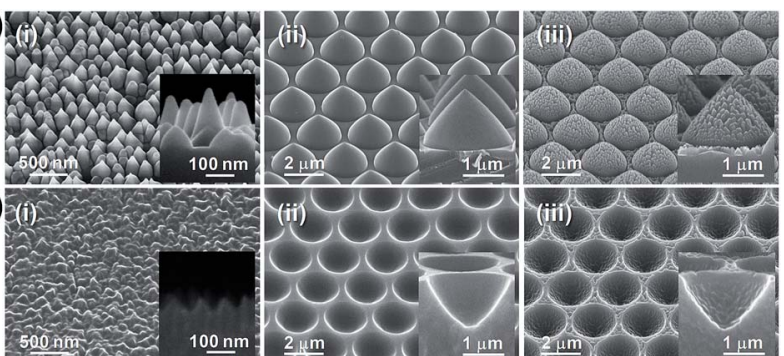

(c) (i)
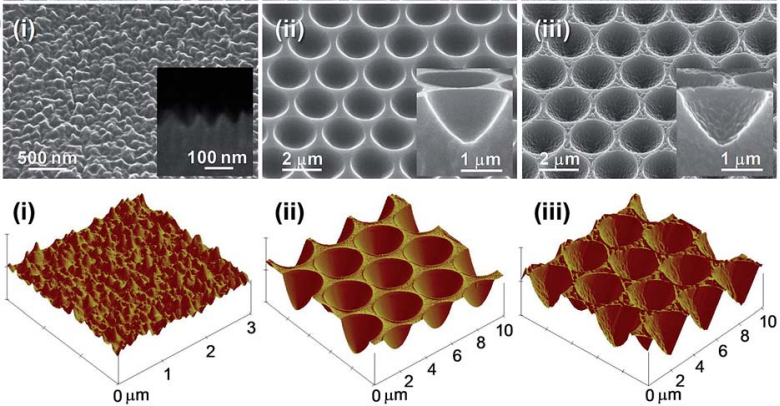

Fig. 2 FE-SEM images of (a) sapphire master molds with (i) flat NTs, (ii) p-MGs, and (iii) CESs and (b) PDMS films with inverted patterns of the corresponding master molds and (c) AFM images of the PDMS films with (i) flat INTs, (ii) p-IMGs, and (iii) ICESs.

antireflection layer with a self-cleaning function. For the samples with different surface morphologies, the water contact angles were measured to investigate the wetting behavior. Fig. 3 shows the photographs of (a) a water droplet on (i) the bare coverglass and the PDMS films with (ii) flat INTs, (iii) p-IMGs, and (iv) ICESs and (b) water droplet cleaning behaviors of (i) the bare coverglass and (ii) the PDMS film with the ICESs. As shown in Fig. 3(a), the bare coverglass had a hydrophilic surface with a water contact angle $\left(\theta_{\mathrm{CA}}\right)$ of $\sim 32^{\circ}$ while the patterned PDMS films showed the $\theta_{\mathrm{CA}}$ values of $>90^{\circ}$ (i.e., hydrophobicity). In particular, the surface of the ICESs PDMS film exhibited the $\theta_{\mathrm{CA}}$ value of $\sim 121^{\circ}$ which is higher than those of the other PDMS films (i.e., $\theta_{\mathrm{CA}} \sim 100^{\circ}$ and $115^{\circ}$ for the PDMS films with the flat INTs and the p-IMGs, respectively). This is ascribed to the increased roughness on the surface of PDMS films with the hydrophobicity, i.e., $\theta_{\mathrm{CA}} \approx 95^{\circ}$ for the flat bare PDMS film (see Fig. S3 in the ESI $\dagger$ ), which can be explained by the Cassie-Baxter theory. ${ }^{41,42}$ This $\theta_{\mathrm{CA}}$ value of $\sim 121^{\circ}$ is relatively lower than those (i.e., $\theta_{\mathrm{CA}}>150^{\circ}$, superhydrophobicity) reported in other previous works. ${ }^{35,42-44}$ However, as can be seen in Fig. 3(b), after dropping the water droplets, the black charcoal particles on the ICESs PDMS film were clearly removed by the rolling down water droplets (i.e., self-cleaning). On the other hand, the black charcoal (or dust) particles on the bare coverglass were just relocated, thus partially remaining with water droplets at the edge of coverglass (see Fig. S4 and Video in the ESI $\dagger$ ). Thus, this ICESs PDMS film with a hydrophobic surface is very useful because an optical interface with an additional self-cleaning effect would enhance the practical feasibility in outdoor environments.

\section{Optical properties}

Fig. 4 shows the measured (a) total and (b) diffuse transmittance spectra of the PDMS films with the flat INTs, the pIMGs, and the ICESs laminated on the coverglasses. For comparison, the total and diffuse transmittance spectra of the (a) (i)

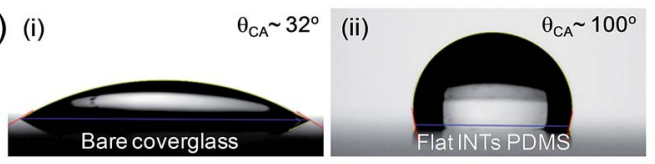

(iii)

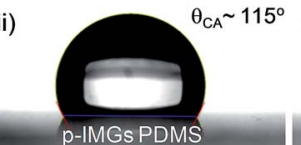

(iv)

(b)

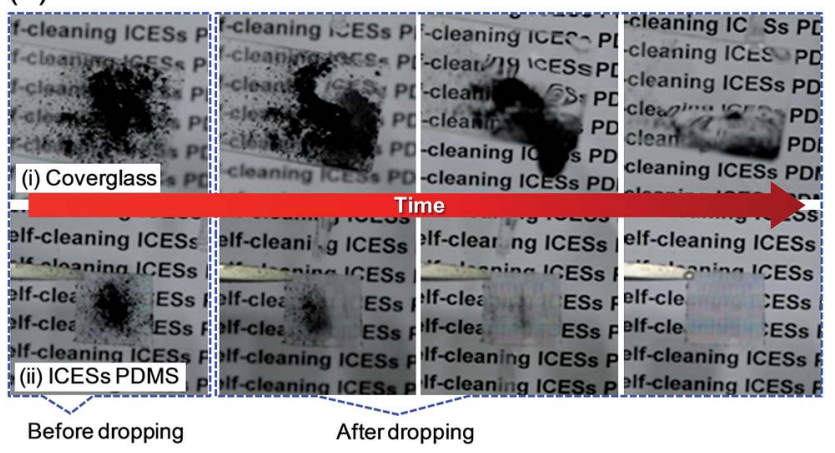

Fig. 3 Photographs of (a) a water droplet on (i) bare coverglass and the PDMS films with (ii) flat INTs, (iii) p-IMGs, and (iv) ICESs and (b) water droplet cleaning behaviors of (i) bare coverglass and (ii) PDMS film with the ICESs. The $\theta_{C A}$ is a water contact angle.

bare coverglass are also shown in Fig. 4(a) and (b), respectively. As shown in Fig. 4(a), by attaching the PDMS film with the flat INTs on the coverglass, the total transmittance was increased compared to the coverglass over a wide wavelength range of $350-900 \mathrm{~nm}$ due to the formation of a linear gradient-refractiveindex profile between air and the PDMS caused by the INTs as well as the step gradient-refractive-index variation in the constituent materials from air $(n=1)$ to the coverglass $(n \approx$ 1.53) via the PDMS $(n \approx 1.4-1.43){ }^{49,50}$ For the p-IMGs PDMS film, on the contrary, the total transmittance spectrum was higher than that of the flat INTs PDMS film. This is the reason why the inverted conical microgratings with a grooved geometry feature have a more linear gradient-refractive-index profile from air to the PDMS due to the large depth of $\sim 1.5 \mu \mathrm{m}$ and also lead to the extension of effective optical path lengths caused by the diffracted and rebounded lights between the inverted microgratings,${ }^{24,51,52}$ which can effectively enhance the transmission in transparent materials by suppressing the surface reflection. By introducing the nanotextures on the p-IMGs (i.e., ICESs), the total transmittance was further increased, exhibiting the average total transmittance ( $T_{\text {avg }}$ ) of $\sim 94.1 \%$ which is a higher value than those of the other samples (i.e., $T_{\text {avg }} \approx 90,90.6$, and 93.3\% for the bare coverglass, the flat INTs PDMS/coverglass, and the p-IMGs PDMS/coverglass, respectively). This can be also explained by the increased trapping of the reflected lights from escaping back to air due to the INTs which have the small period of $\sim 100 \mathrm{~nm}$ and the effective homogeneous medium with a gradient-refractive-index profile. Therefore, the ICESs can further reduce the reflection or enhance the transmission. ${ }^{16-26}$ The light diffraction behaviors in these samples 
will be covered in the paragraph of Fig. 4(b). For solar cell applications, the solar weighted transmittance (SWT) was estimated using a well-known equation: ${ }^{53}$

$$
\mathrm{SWT}=\frac{\int_{350 \mathrm{~nm}}^{900 \mathrm{~nm}} F(\lambda) T(\lambda) \mathrm{d} \lambda}{\int_{350 \mathrm{~nm}}^{900 \mathrm{~nm}} F(\lambda) \mathrm{d} \lambda},
$$

where $F(\lambda)$ is a spectral photon irradiance (i.e., air mass 1.5 global $)^{54}$ and $T(\lambda)$ is the total transmittance in Fig. 4(a). As expected, the ICESs PDMS/coverglass had the higher SWT value of $\sim 94.3 \%$ compared to the other samples (i.e., SWT $\approx 90.2$, 90.8, and 93.4\% for the bare coverglass, the flat INTs PDMS/ coverglass, and the p-IMGs PDMS/coverglass, respectively). For all the samples, the total reflectance spectra were also investigated, as shown in the inset of Fig. 4(a). The ICESs PDMS film considerably reduced the reflectance of coverglass over a wide wavelength range of 350-900 $\mathrm{nm}$, exhibiting the lower solar weighted reflectance (SWR) value of $\sim 5.1 \%$ than those of the other samples (i.e., SWR $\approx 9.2,8.3$, and 5.7\% for the bare coverglass, the flat INTs PDMS/coverglass, and the p-IMGs PDMS/ coverglass, respectively). Herein, the SWR can be also evaluated from the SWT formula in eqn (1) by replacing the transmittance $(T)$ with the reflectance $(R)$. Many studies on antireflective nanostructures with small dimensions (i.e., period, diameters, and heights) of 200-250 $\mathrm{nm}$ for glasses or polymers (e.g., PDMS, polyurethane, and polymethylmethacrylate) with a flat surface have reported the reduction of $\sim 3-4 \%$ in the reflection. ${ }^{43,55-61}$ Whereas, the flat INTs PDMS film in this work showed a relatively poor antireflection effect (i.e., reduction of $\sim 0.9 \%$ in the SWR) due to its low depth of $\sim 90 \pm 10 \mathrm{~nm}$. If the INTs PDMS film has the depths larger than $200-250 \mathrm{~nm}$, it would also decrease the reflectance of bare coverglass with a reduction of $\sim 3-4 \% .^{55,57,61}$ However, this low depth of INTs is caused by the limitations due to the relatively small size and thin thickness of the thermally dewetted Au nanopatterns as the etch mask of master molds for the Au film thickness of $4 \mathrm{~nm}$ in this experiment to obtain the tapered NTs with periods of $<200 \mathrm{~nm}$ which could be fabricated using the overall etching until the Au nanoparticles were completely removed. On the contrary, the nanostructures prepared by the thicker metal films have a poor transmission at short wavelengths because of the diffraction losses though they have heights (or depths) larger than 200-250 $n m .^{55,57}$ Therefore, to achieve the desirable nanostructures on the flat surface of polymer films for efficient antireflection, it is necessary to further study for the optimization of master molds in fabrication process conditions such as the nanopattering using thermally-dewetted dot-like metal nanoparticles (e.g., metal film thickness, heating temperature, heat-treatment time, etc.) and dry etching (i.e., RF power, additional ICP power, process pressure, etching time, gas flow rate, etc.). Additionally, for this ICESs PDMS film, the improvement in transmittance (or antireflection) properties can be verified in the photograph of Fig. S5 of the ESI. $\uparrow$ For the bare coverglass, the characters under it are nearly not seen due to the strongly reflected white fluorescent light at the surface. On the other hand, the ICESs PDMS
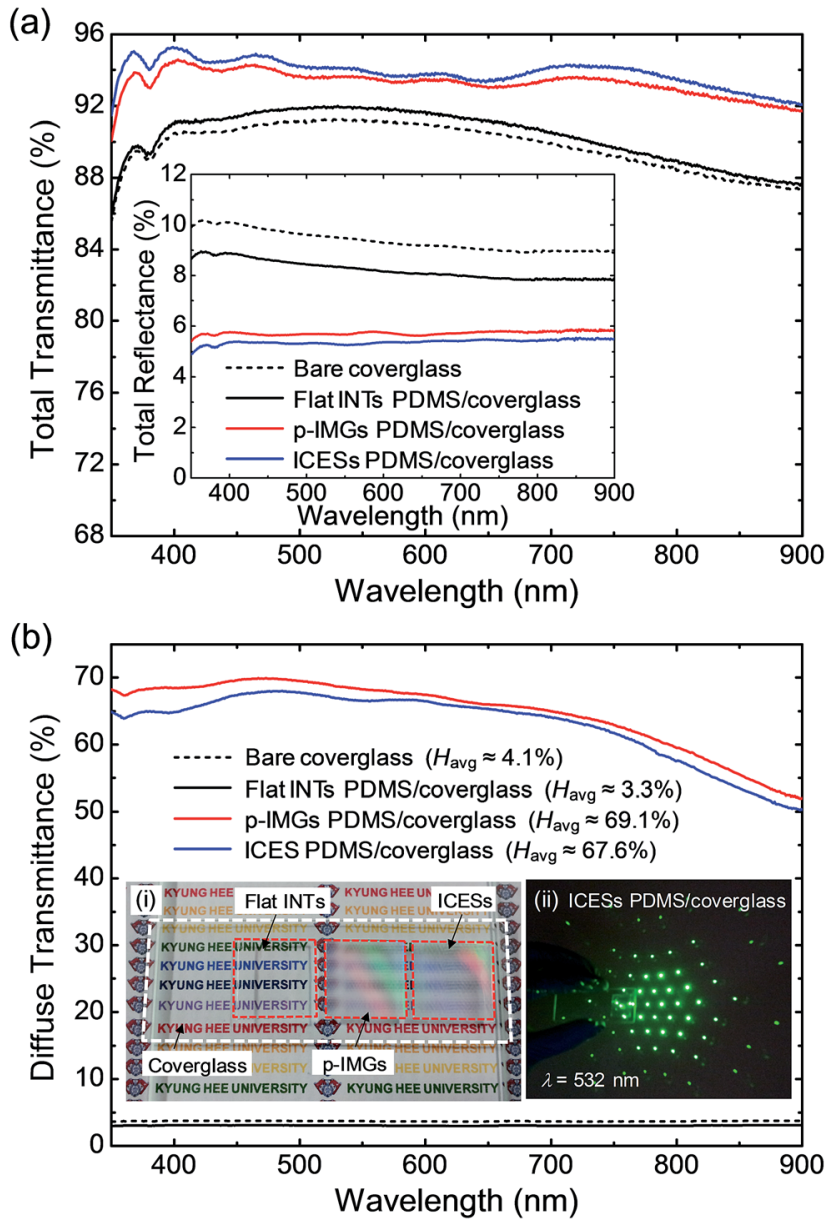

(c)
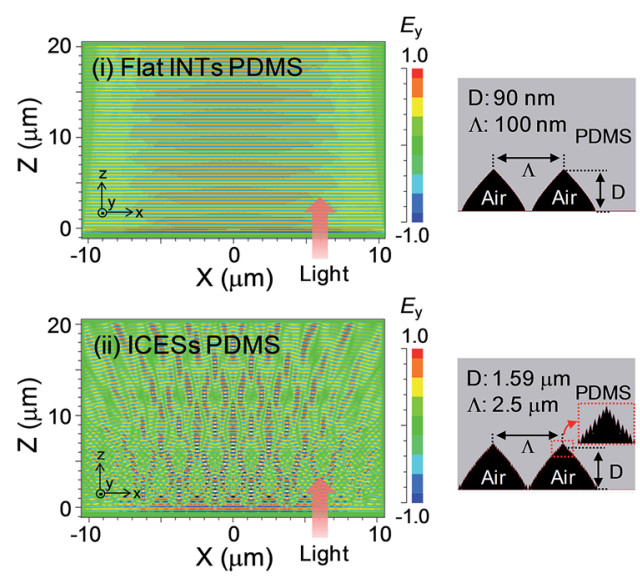

Fig. 4 Measured (a) total and (b) diffuse transmittance spectra of the PDMS films with the flat INTs, the p-IMGs, and the ICESs laminated on the coverglasses and (c) contour plots of calculated electric field distributions by FDTD simulations for the incident light propagating from air to the PDMS films with (i) the flat INTs and (ii) the ICESs and scale-modified corresponding simulation models. For comparison, the total and diffuse transmittance spectra of the bare coverglass are also shown in (a) and (b), respectively. Inset of (a) shows the total reflectance spectra of the corresponding samples. Photographs of (i) PDMS films with the flat INTs, the p-IMGs, and the ICESs (red-dotted lines) laminated on the coverglass (white-dotted line) and (ii) diffraction phenomenon of the ICESS PDMS/coverglass using a diode laser with $\lambda=532 \mathrm{~nm}$ are shown in the insets of (b), respectively. 
film/coverglass (red-dotted line) exhibits a better legibility for the characters below the sample though the characters are hazy.

For a periodic grating structure, when a light enters into the grating with a period of $\Lambda$ at normal incidence, the angle of the transmitted diffraction waves, $\theta_{\mathrm{t}, m}$, in the $m$-th diffraction order is given by the grating equation: ${ }^{62}$

$$
\sin \theta_{\mathrm{t}, m}=\frac{m \lambda}{\Lambda n},
$$

where $\lambda$ is the wavelength of incident light and $n$ is the refractive index of the incident medium. As mentioned above, for the nanograting structures (such as NTs) with periods lower than $\sim 200 \mathrm{~nm}$ on the transparent medium, the high transmittance can be obtained without diffraction losses, which are strongly dependent on the period of structures, at visible and NIR wavelengths due to the only allowed zeroth order diffracted light wave in the transmission. ${ }^{55,57,63}$ On the other hand, the grating structures with periods larger than incident wavelengths generate higher order diffraction lights which would decrease the transmission. However, for the transparent materials with low refractive indices including glasses, quartzes, sapphires, polymers, etc., the cone-shaped structures with desirable microscale periods can lead to the high total and diffuse transmittances, simultaneously. ${ }^{23-25}$ Therefore, the hierarchical structure consisting of NTs and MGs, i.e., CESs, can further increase both the total and diffuse transmittances. As shown in Fig. 4(b), both the p-IMGs and ICESs PDMS films on coverglasses have much higher diffuse transmittance spectra over a wide wavelength region of 350-900 $\mathrm{nm}$ while there are almost no diffracted lights for the bare coverglass and the flat INTs PDMS/coverglass. However, the diffuse transmittance of ICESs PDMS/coverglass was slightly reduced compared to the p-IMGs PDMS/coverglass due to the additional nanotextured surface with a low dimension of $\sim 100$ nm-period on p-IMGs PDMS film, which suppresses the light scattering and enhances the light traveling straight. ${ }^{25}$ The haze ratio $(H)$, which is defined by the ratio of the diffuse $\left(T_{\mathrm{d}}\right)$ to the total $\left(T_{\mathrm{t}}\right)$ transmission, i.e., $H(\%)=T_{\mathrm{d}} / T_{\mathrm{t}} \times$ 100 , is often used to characterize the light scattering properties of a sample. For both the p-IMGs PDMS/coverglass and ICESs PDMS/coverglass, the average $H\left(H_{\text {avg }}\right)$ values were estimated to be $\sim 69.1$ and $67.6 \%$ at wavelengths of $350-900 \mathrm{~nm}$, respectively, (i.e., $H_{\mathrm{avg}} \approx 4.1$ and $3.3 \%$ for the bare coverglass and the flat INTs PDMS/coverglass, respectively). In the transmission, these high diffraction behaviors (i.e., light scattering) can be confirmed in the insets in Fig. 4(b). The photographs of (i) PDMS films with the flat INTs, the p-IMGs, and the ICESs (red-dotted lines) laminated on the coverglass (white-dotted line) and (ii) diffraction phenomenon of the ICESs PDMS/coverglass using a diode laser with $\lambda=532 \mathrm{~nm}$ are shown in the insets of Fig. 4(b), respectively. In Fig. 4(b)(i), the characters below the bare coverglass and the flat INTs PDMS/coverglass are well seen. On the other hand, for the p-IMGs PDMS/coverglass and the ICESs PDMS/coverglass, the characters below both the samples are not relatively distinguished due to the strong light scattering. In addition, for the ICESs PDMS/coverglass of Fig. 4(b)(ii), high order diffraction patterns in transmitted lights for $\lambda=532 \mathrm{~nm}$ are shown. The contour plots of calculated electric field distributions for the incident light propagating from air to the PDMS films with (i) the flat INTs and (ii) the ICESs and the scalemodified corresponding simulation models used in these calculations are shown in Fig. 4(c). The simulation results indicate that micron-based ICESs, i.e., period $(\Lambda)=2.5 \mu \mathrm{m}$, exhibit strong light interference patterns with a wide angular spread and help the light propagation across the interface between air and the PDMS while there are no significant scattering lights for the flat INTs due to the subwavelength-scale of $100 \mathrm{~nm} .{ }^{24}$ From these results, it is noted that the ICESs PDMS film enables to increase the diffuse transmittance of the coverglass, maintaining the high total transmission, and thus it can lead to the PCE improvement of encapsulated solar cells because of the enhanced light harvesting in the absorption layer of devices caused by the light scattering and antireflection effects. ${ }^{20-23}$

\section{Device feasibility}

For practical device applications, all the PDMS films were employed onto the coverglass of encapsulated GaAs singlejunction solar cells. Fig. 5 shows (a) EQE spectra and (b) current density-voltage $(J-V)$ curves of encapsulated III-V GaAs single-junction solar cells with the flat INTs, the p-IMGs, and the ICESs PDMS films laminated on coverglasses. For comparison, the EQE spectrum and $J-V$ curve of encapsulated III-V GaAs single-junction solar cell with the bare coverglass are also shown in Fig. 5(a) and (b), respectively. The measured device characteristics (i.e., open circuit voltage; $V_{\mathrm{oc}}$, short circuit current density; $J_{\text {sc }}$, fill factor; FF, PCE) of encapsulated GaAs single-junction solar cells with different antireflection PDMS layers of coverglass are summarized in the inset of Fig. 5(b). As shown in Fig. 5(a), by attaching the PDMS films to the coverglass of encapsulated solar cell devices, the increased EQE spectra were obtained compared to the device with the bare coverglass. The device with the ICESs PDMS/coverglass exhibited improved EQE values compared to the other devices at wavelengths of $400-870 \mathrm{~nm}$ due to the larger photogenerated carriers caused by its higher total transmittance and haze properties. As can be seen in Fig. 5(b), compared to the device with the bare coverglass, the use of PDMS films on the coverglass of encapsulated solar cells definitely caused an enhancement of $J_{\text {sc }}$ values while having similar $V_{\text {oc }}$ and FF values, and thus it enhanced the PCE of encapsulated solar cells. In particular, for the device with the ICESs PDMS/coverglass, the higher $J_{\mathrm{sc}}$ value of $27.64 \mathrm{~mA} \mathrm{~cm}^{-2}$ was obtained than that (i.e., $J_{\mathrm{sc}}=26 \mathrm{~mA} \mathrm{~cm}^{-2}$ ) of the device with the bare coverglass, as previously demonstrated by EQE measurements. This is attributed to the dramatically increased $H_{\text {avg }}$ value of $\sim 67.6 \%$ and SWT value of $\sim 94.3 \%$ over a wide wavelength range of 350-900 $\mathrm{nm}$, which leads to the larger photocurrents due to the enhanced light harvesting in the underneath device cell absorption layer. The increase in $J_{\mathrm{sc}}$ is the main reason of the PCE improvement from 22.96 to $24.3 \%$, indicating the PCE increment percentage of $\sim 5.8 \%$.

To demonstrate the efficient light-harvesting function (i.e., antireflection and light scattering) of the ICESs PDMS film on the device performance under obliquely incident sunlight, for the corresponding encapsulated III-V GaAs single-junction 

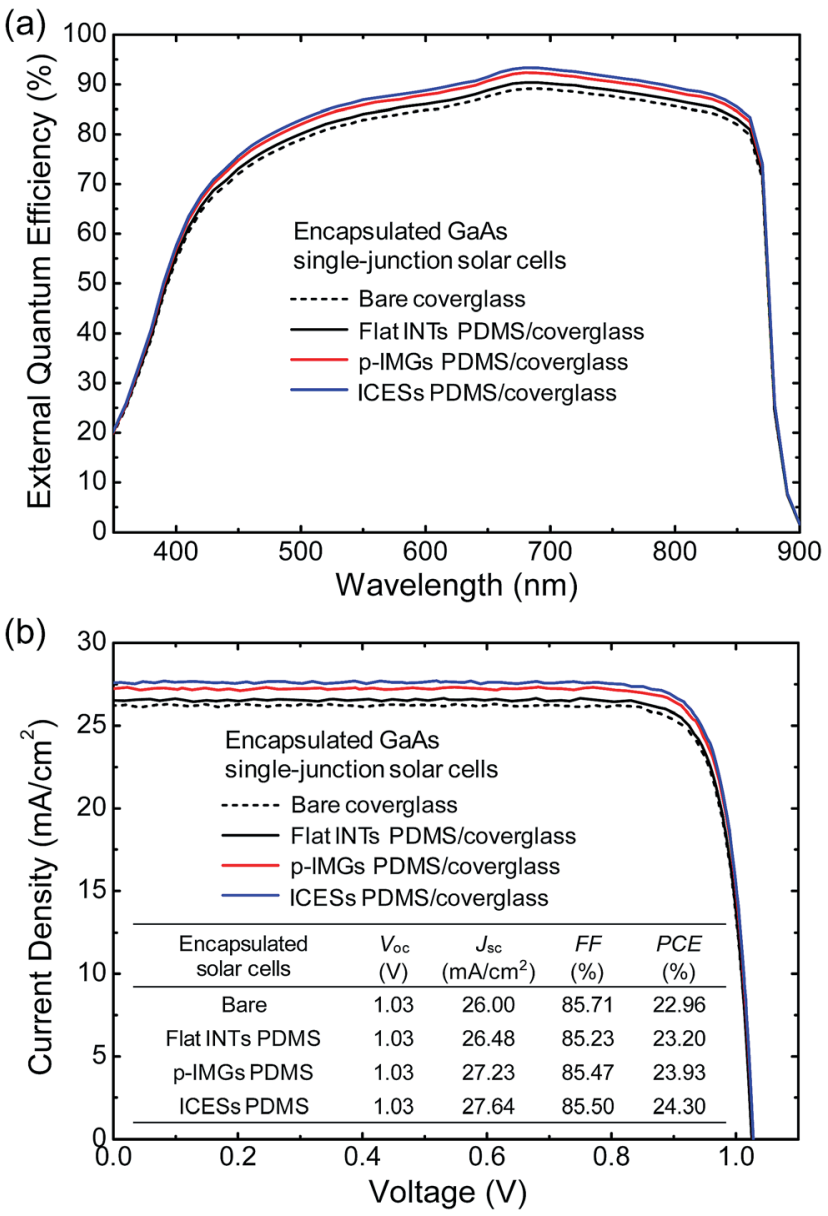

Fig. 5 (a) EQE spectra and (b) J-V curves of encapsulated III-V GaAs single-junction solar cells with the flat INTs, the p-IMGs, and the ICESs PDMS films laminated on coverglasses. For comparison, EQE spectrum and $J-V$ curve of encapsulated III-V GaAs single-junction solar cell with the bare coverglass are also shown in (a) and (b), respectively. Measured device characteristics of encapsulated GaAs single-junction solar cells with different antireflection PDMS layers of coverglass are summarized in the inset of (b).

solar cells, their $J_{\mathrm{sc}}$ values were measured and the increment percentage in $J_{\mathrm{sc}}$ relative to the device with the bare coverglass was estimated at incident angles $\left(\theta_{\text {In }}\right)$ of $20-70^{\circ}$, as shown in Fig. 6. For incident light angle-dependent $J-V$ measurements, inclined mounts with tilting angles of $20-70^{\circ}$ were used. As the $\theta_{\text {In }}$ was increased from $20^{\circ}$ to $70^{\circ}$, the $J_{\mathrm{sc}}$ values of all the devices were generally decreased, which shows a similar trend with the other previous reports. ${ }^{11,13,64,65}$ This is ascribed to the reduction of the projection area where the incident light enters into the device due to the tilted device from the normally incident light source of the solar simulator as well as the increased surface reflection losses. ${ }^{11,65}$ However, the device with the ICESs PDMS/ coverglass showed the higher $J_{\mathrm{sc}}$ value at each $\theta_{\mathrm{In}}$, keeping a larger average increment percentage of $\sim 9.2 \%$ relative to the device with the bare coverglass at $\theta_{\text {In }}=20-70^{\circ}$ (i.e., $\sim 3.4 \%$ and $\sim 6.7 \%$ for the flat INTs PDMS/coverglass and the p-IMGs PDMS/ coverglass, respectively). Thus, the use of the ICESs DPMS film as an antireflective protective layer could improve the solar

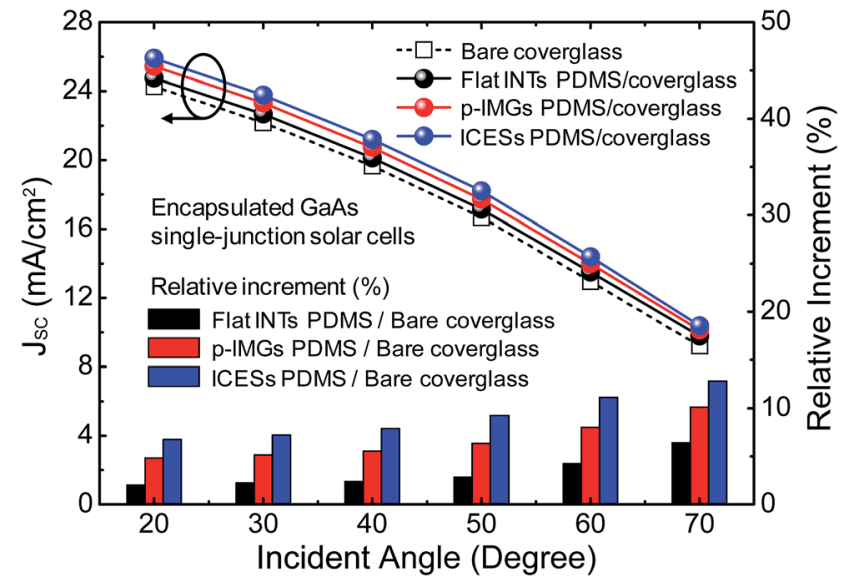

Fig. $6 J_{\mathrm{sc}}$ values of the encapsulated III-V GaAs single-junction solar cells with the bare coverglass and the flat INTs, the p-IMGs, and the ICESs PDMS films laminated on coverglasses and estimated increment percentage in $J_{s c}$ for the devices with the flat INTs, the $p$-IMGs, and the ICESs PDMS films relative to the device with the bare coverglass at $\theta_{\ln }=20-70^{\circ}$.

power generation in encapsulated photovoltaic systems for an entire day and the seasons.

\section{Self-cleaning effect and stability on device performance under external environment}

To investigate the self-cleaning function of the ICESs PDMS film on the device performance, the encapsulated solar cells were exposed in outdoor environment. Fig. 7 shows the (a) SWT values of the bare coverglass and the ICESs PDMS/coverglass and (b) $J_{\mathrm{sc}}$ and PCE values of corresponding encapsulated III$\mathrm{V}$ GaAs single-junction solar cells to show the self-cleaning effect. The photographs of the bare coverglass and the ICESs PDMS/coverglass covered by dusts for 7 days under external environment before and after cleaning by falling raindrops are shown in the inset of Fig. 7(a). The samples were mounted on the holder with about $30^{\circ}$ tilting angle at the rooftop of a building. For 6 days, there was little rain, and then it rained on the 7th day. As shown in Fig. 7(a), the SWT values of all the samples covered by dusts were reduced from $\sim 90.2$ and $\sim 94.3 \%$ to $\sim 88.1$ and $\sim 92.2 \%$ for the bare coverglass and the ICESs PDMS/coverglass, respectively, because of the interrupting of incident light by the dusts. However, after the cleaning by the falling raindrops, the SWT value of the ICESs PDMS/coverglass was increased by $\sim 93.6 \%$, exhibiting the larger recovery percentage of approximately $1.6 \%$ compared to that (i.e., $\sim 1.2 \%$ ) of the bare coverglass (i.e., SWT $\approx 89.2 \%$ ) in the transmittance. As verified in the photographs of Fig. 7(a), after the cleaning, the dust blotches remained over an entire surface of the bare coverglass while the surface of the ICESs PDMS film was nearly clear due to its self-cleaning effect caused by the hydrophobicity (i.e., $\theta_{\mathrm{CA}} \approx 121^{\circ}$ ). In Fig. $7(\mathrm{~b})$, similarly, the dusts blocked the incident light into the devices, and thus $J_{\text {sc }}$ values of all the devices were also decreased with a reduction in their PCE values (i.e., from $J_{\mathrm{sc}}=26$ and $27.64 \mathrm{~mA} \mathrm{~cm}{ }^{-2}$ and PCE $=22.96$ and $24.3 \%$ to $J_{\mathrm{sc}}=25.4$ and $27.03 \mathrm{~mA} \mathrm{~cm}{ }^{-2}$ and PCE $=$ 
22.44 and $23.76 \%$ for the devices with the bare coverglass and the ICESs PDMS/coverglass, respectively). However, after the cleaning, the $J_{\mathrm{sc}}$ and PCE values of the device with the ICESs PDMS/coverglass were further enhanced to be $27.44 \mathrm{~mA} \mathrm{~cm}^{-2}$ and $24.13 \%$, respectively, showing a superior PCE recovery percentage of $\sim 1.6 \%$ compared to the device with the bare coverglass (i.e., $\sim 1.2 \%$ recovery percentage, $J_{\mathrm{sc}}=25.71 \mathrm{~mA}$ $\mathrm{cm}^{-2}, \mathrm{PCE}=22.7 \%$ ). Unlike the $J_{\mathrm{sc}}$, on the other hand, the $V_{\mathrm{oc}}$ and $\mathrm{FF}$ values were less influenced by the change of the transmittance due to the dusts. ${ }^{35,48}$

Additionally, to evaluate the self-cleaning function on the long-term device stability of the encapsulated solar cells under external environment, their device performance was further explored for approximately one month. Fig. 8 shows (a) SWT values of the bare coverglass and the ICESs PDMS/coverglass and (b) $J_{\mathrm{sc}}$ values (c) PCE values of the corresponding encapsulated III-V GaAs single-junction solar cells to show the selfcleaning effect for 32 days under external environment. As
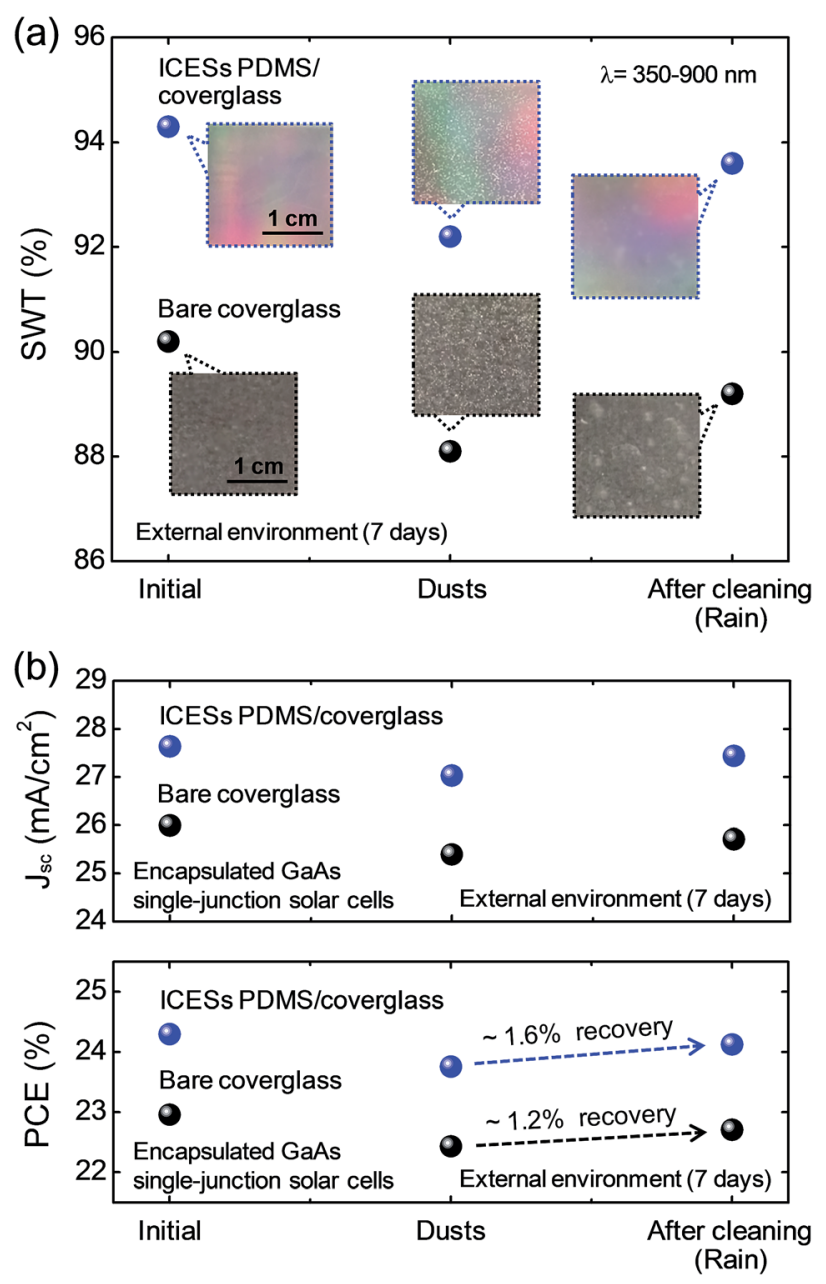

Fig. 7 (a) SWT values of the bare coverglass and the ICESS PDMS/ coverglass and (b) $J_{S C}$ and PCE values of the corresponding encapsulated III-V GaAs single-junction solar cells to show the self-cleaning effect. Photographs of the bare coverglass and the ICESS PDMS/coverglass covered by dusts for 7 days under external environment before and after cleaning by falling raindrops are shown in the inset of (a).
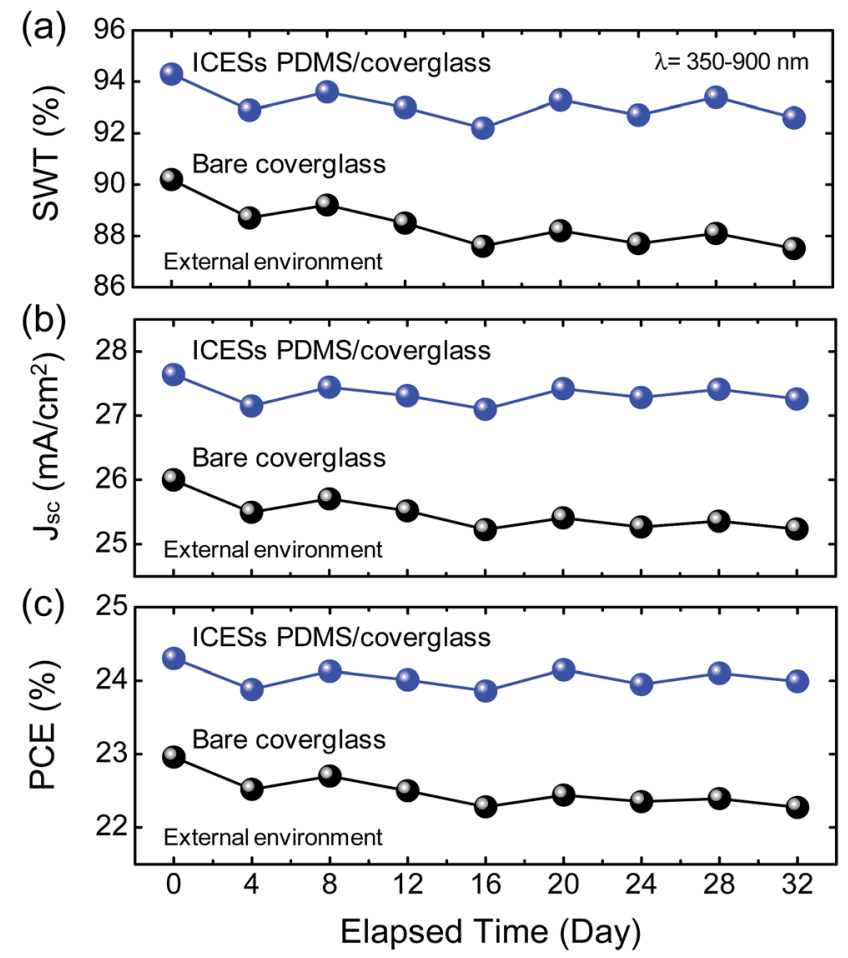

Fig. 8 (a) SWT values of the bare coverglass and the ICESS PDMS/ coverglass and (b) $J_{\mathrm{sc}}$ values (c) PCE values of the corresponding encapsulated III-V GaAs single-junction solar cells to show the longterm self-cleaning effect for 32 days under external environment.

expected from Fig. 8, the $J_{\mathrm{sc}}$ intensively depended on the SWT, and thus the PCE mainly varied as it. For the device with the bare coverglass, its performance was generally degraded after 32 days with decreasing the SWT, showing the drop percentages of $\sim 2.9 \%$ and $\sim 3 \%$ in $J_{\text {sc }}$ and PCE, respectively, compared to the initial values. On the contrary, owing to the good self-cleaning function of the ICESs PDMS film, the ICESs PDMS/coverglass showed an average increment percentage of $\sim 5.3 \%$ compared to the bare coverglass in the SWT for 32 days. As a result, the device with the ICESs PDMS/coverglass exhibited the superior long-term stability with the lower drop percentages of $\sim 1.3 \%$ and $\sim 1.4 \%$ in $J_{\mathrm{sc}}$ and PCE, respectively.

\section{Conclusions}

We fabricated the highly-transparent and hazy ICESs PDMS films with antireflective and hydrophobic properties for efficient light-harvesting and self-cleaning effects for encapsulated III-V GaAs single-junction solar cell applications. The ICESs PDMS film was fabricated by a facile, simple, and cost-effective soft lithography method using sapphire mater molds with CESs consisting of hierarchical NTs/p-MGs. The ICESs PDMS film exhibited larger $\theta_{\mathrm{CA}} \approx 121^{\circ}$, SWT $\approx 94.3 \%$, and $H_{\mathrm{avg}} \approx 67.6 \%$, respectively, than those (i.e., $\theta_{\mathrm{CA}} \approx 32^{\circ}$, SWT $\approx 90.2 \%$, and $H_{\text {avg }}$ $\approx 4.1 \%$, respectively) of the bare coverglass. By laminating the ICESs PDMS film on the coverglass in the encapsulated III-V GaAs single-junction solar cell, the increased $J_{\mathrm{sc}}$ value of 27.64 $\mathrm{mA} \mathrm{cm}{ }^{-2}$ was obtained compared to the encapsulated solar cell 
with the bare coverglass (i.e., $J_{\mathrm{sc}}=26 \mathrm{~mA} \mathrm{~cm}^{-2}$ ), and this mainly enhanced the PCE by $24.3 \%$ (i.e., PCE $=22.96 \%$ for the encapsulated cell with the bare coverglass), exhibiting the PCE increment percentage of $\sim 5.8 \%$. Furthermore, it also showed not only superior device performance at obliquely incident lights, but also good long-term device stability with the only PCE drop percentage of $\sim 1.4 \%$ after 32 days. These results suggest that the artificial inverted compound eye structured polymer films, which can be easily fabricated by the simple and costeffective soft lithography technique, with the high optical transparency and haze as well as the hydrophobic surface have considerable potential for high-performance and long-term stability photovoltaic systems using transparent substrates as well as coverglasses in terms of efficient light-harvesting and self-cleaning functions.

\section{Acknowledgements}

This work was supported by the National Research Foundation of Korea (NRF) grant funded by the Korea government (MSIP) (No. 2014-069441). The authors would like to thank the Korea Advanced Nano-Fab Center (KANC) for providing the solar cells.

\section{References}

1 P. Yu, C. H. Chang, C. H. Chiu, C. S. Yang, J. C. Yu, H. C. Kuo, S. H. Hsu and Y. C. Chang, Adv. Mater., 2009, 21, 1618.

2 J. W. Leem, X. Y. Guan, M. Choi and J. S. Yu, Sol. Energy Mater. Sol. Cells, 2015, 134, 45.

3 D. J. Aiken, Sol. Energy Mater. Sol. Cells, 2000, 64, 393.

4 Y. M. Song, Y. Jeong, C. I. Yeo and Y. T. Lee, Opt. Express, 2012, 20, A916.

5 M. A. Green, K. Emery, Y. Hishikawa, W. Warta and E. D. Dunlop, Prog. Photovoltaics, 2014, 22, 1.

6 P. Kuang, J. M. Park, W. Leung, R. C. Mahadevapuram, K. S. Nalwa, T. G. Kim, S. Chaudhary, K. M. Ho and K. Constant, Adv. Mater., 2011, 23, 2469.

7 H. Nagel, A. G. Aberle and R. Hezel, Prog. Photovoltaics, 1999, 7, 245.

8 S. M. Yang, Y. C. Hsieh and C. A. Jeng, J. Vac. Sci. Technol., A, 2009, 27, 336.

9 P. Lalanne and G. M. Morris, Proc. SPIE, 1996, 2776, 300.

10 N. Kadakia, S. Naczas, H. Bakhru and M. Huang, Appl. Phys. Lett., 2010, 97, 191912.

11 C. I. Yeo, H. J. Choi, Y. M. Song, S. J. Kang and Y. T. Lee, J. Mater. Chem. A, 2015, 3, 7235.

12 Y. Nasuno, N. Kohama, K. Nishimura, T. Hayakawa, H. Taniguchi and M. Shimizu, Appl. Phys. Lett., 2006, 88, 071909.

13 J. W. Leem, M. Choi and J. S. Yu, ACS Appl. Mater. Interfaces, 2015, 7, 2349.

14 S. Yang, Y. Wang, L. Bai, B. Liu, J. Fan, X. Yang, H. Zhao, C. Wei, Q. Huang, X. Chen, G. Wang, Y. Zhao and X. Zhang, RSC Adv., 2013, 3, 208.

15 S. Y. Han, C. Pan, D. H. Kim and C. H. Chang, RSC Adv., 2015, 5, 24712.
16 J. W. Leem, Y. M. Song and J. S. Yu, Nanoscale, 2013, 5, 10455.

17 G. Li, J. Li, C. Zhang, Y. Hu, X. Li, J. Chu, W. Huang and D. Wu, ACS Appl. Mater. Interfaces, 2015, 7, 383.

18 R. J. Martín-Palma, C. G. Pantano and A. Lakhtakia, Nanotechnology, 2008, 19, 355704.

19 H. K. Raut, S. S. Dinachali, Y. C. Loke, R. Ganesan, K. K. Ansah-Antwi, A. Góra, E. H. Khoo, V. A. Ganesh, M. S. M. Saifullah and S. Ramakrishna, ACS Nano, 2015, 9, 1305.

20 C. A. Lin, K. Yu Lai, W. C. Lien and J. H. He, Nanoscale, 2012, 4, 6520.

21 C. H. Ho, D. H. Lien, H. C. Chang, C. A. Lin, C. F. Kang, M. K. Hsing, K. Y. Lai and J. H. He, Nanoscale, 2012, 4, 7346.

22 Y. Liu, A. Das, S. Xu, Z. Lin, C. Xu, Z. L. Wang, A. Rohatgi and C. P. Wong, Adv. Energy Mater., 2012, $2,47$.

23 B. Janthong, Y. Moriya, A. Hongsingthong, P. Sichanugrist and M. Konagai, Sol. Energy Mater. Sol. Cells, 2013, 119, 209.

24 Y. H. Ko and J. S. Yu, Opt. Express, 2011, 19, 15574.

25 J. W. Leem, M. S. Kim and J. S. Yu, J. Opt. Soc. Am. B, 2013, 30, 1665.

26 Y. P. Chen, C. H. Lee and L. A. Wang, Nanotechnology, 2011, 22, 215303.

27 K. H. Li, C. Feng and H. W. Choi, Appl. Phys. Lett., 2014, 104, 051107.

28 S. H. Huang, R. H. Horng, K. S. Wen, Y. F. Lin, K. W. Yen and D. S. Wuu, IEEE Photonics Technol. Lett., 2006, 18, 2623.

29 R. H. Kim, D. H. Kim, J. Xiao, B. H. Kim, S. I. Park, B. Panilaitis, R. Ghaffari, J. Yao, M. Li, Z. Liu, V. Malyarchuk, D. G. Kim, A. P. Le, R. G. Nuzzo, D. L. Kaplan, F. G. Omenetto, Y. Huang, Z. Kang and J. A. Rogers, Nat. Mater., 2010, 9, 929.

30 N. Koo, M. Bender, U. Plachetka, A. Fuchs, T. Wahlbrink, J. Bolten and H. Kurz, Microelectron. Eng., 2007, 84, 904.

31 Y. M. Song, Y. Xie, V. Malyarchuk, J. Xiao, I. Jung, K. J. Choi, Z. Liu, H. Park, C. Lu, R. H. Kim, R. Li, K. B. Crozier, Y. Huang and J. A. Rogers, Nature, 2013, 497, 95.

32 J. W. Leem, S. Kim, S. H. Lee, J. A. Rogers, E. Kim and J. S. Yu, Adv. Energy Mater., 2014, 4, 1301315.

33 J. H. Koschwanez, R. H. Carlson and D. R. Meldrum, PLoS One, 2009, 4, 4572.

34 S. Al-Maawali, J. E. Bemis, B. B. Akhremitchev, R. Leecharoen, B. G. Janesko and G. C. Walker, J. Phys. Chem. B, 2001, 105, 3965.

35 Y. B. Park, H. Im, M. Im and Y. K. Choi, J. Mater. Chem., 2011, 21, 633.

36 J. C. Lötters, W. Olthuis, P. H. Veltink and P. Bergveld, J. Micromech. Microeng., 1997, 7, 145.

37 Q. Chen, G. Li, Y. Nie, S. H. Yao and J. L. Zhao, Microfluid. Nanofluid., 2014, 16, 83.

38 C. J. Ting, F. Y. Chang, C. F. Chen and C. P. Chou, J. Micromech. Microeng., 2008, 18, 075001.

39 S. H. Ahn and L. J. Guo, ACS Nano, 2009, 3, 2304.

40 M. A. G. Lazo, R. Teuscher, Y. Leterrier, J. A. E. Månson,

C. Calderone, A. Hessler-Wyser, P. Couty, Y. Ziegler and

D. Fischer, Sol. Energy Mater. Sol. Cells, 2012, 103, 147. 
41 A. B. D. Cassie and S. Baxter, Trans. Faraday Soc., 1944, 40, 546.

42 A. D. Tserepi, M. E. Vlachopoulou and E. Gogolides, Nanotechnology, 2006, 17, 3977.

43 K. C. Park, H. J. Choi, C. H. Chang, R. E. Cohen, G. H. McKinley and G. Barbastathis, ACS Nano, 2012, 5, 3789.

44 W. K. Cho and I. S. Choi, Adv. Funct. Mater., 2008, 18, 1089.

45 J. W. Leem and J. S. Yu, Opt. Express, 2012, 20, 26160.

46 T. W. Odom, J. C. Love, D. B. Wolfe, K. E. Paul and G. M. Whitesides, Langmuir, 2002, 18, 5314.

47 FullWAVE, RSoft Design Group, http:// www.rsoftdesign.com, accessed January 2015.

48 J. Son, S. Kundu, L. K. Verma, M. Sakhuja, A. J. Danner, C. S. Bhatia and H. Yang, Sol. Energy Mater. Sol. Cells, 2012, 98, 46.

49 J. W. Leem, D. H. Joo and J. S. Yu, Sol. Energy Mater. Sol. Cells, 2011, 95, 2221.

50 D. G. Stavenga, S. Foletti, G. Palasantzas and K. Arkawa, Proc. R. Soc. London, Ser. B, 2006, 273, 661.

51 Y. M. Song, G. C. Park, S. J. Jang, J. H. Ha, J. S. Yu and Y. T. Lee, Opt. Express, 2011, 19, A157.

52 J. Zhao and M. A. Green, IEEE Trans. Electron Devices, 1991, 38, 1925.

53 H. Cui, S. Pillai, P. Campbell and M. Green, Sol. Energy Mater. Sol. Cells, 2013, 109, 233.
54 NREL's Renewable Resource Data Center, http:// rredc.nrel.gov/solar/spectra/am1.5, accessed June, 2014.

55 J. W. Leem, Y. Yeh and J. S. Yu, Opt. Express, 2012, 20, 4056. 56 Y. Kanamori, H. Kikuta and K. Hane, Jpn. J. Appl. Phys., 2000, 39, L735.

57 Y. M. Song, H. J. Choi, J. S. Yu and Y. T. Lee, Opt. Express, 2010, 18, 13063.

58 W. L. Min, B. Jiang and P. Jiang, Adv. Mater., 2008, 20, 3914.

59 J. H. Shin, K. S. Han and H. Lee, Prog. Photovoltaics, 2011, 19, 339.

60 D. H. Ko, J. R. Tumbleston, K. J. Henderson, L. E. Euliss, J. M. DeSimone, R. Lopez and E. T. Samulski, Soft Matter, 2011, 7, 6404.

61 K. Choi, S. H. Park, Y. M. Song, Y. T. Lee, C. K. Hwangbo, H. Yang and H. S. Lee, Adv. Mater., 2010, 22, 3713.

62 E. Hecht, Optics, Addison-Wesley, NY, USA, 4th edn, 2002, ch. 10 .

63 J. W. Leem, Y. M. Song and J. S. Yu, Opt. Express, 2011, 19, 26308.

64 C. H. Chang, M. H. Hsu, P. C. Tseng, P. Yu, W. L. Chang, W. C. Sun and W. C. Hsu, Opt. Express, 2011, 19, A219.

65 Y. M. Song, J. H. Jang, J. C. Lee, E. K. Kang and Y. T. Lee, Sol. Energy Mater. Sol. Cells, 2012, 101, 73. 